\title{
Robust Recurrent Cerebellar Model Articulation Controller for Non-Linear MIMO Systems
}

\author{
Xuan-Kien Dang ${ }^{1}$ \\ Ho Chi Minh University of Transport \\ Ho Chi Minh City, Vietnam
}

\author{
Van-Phuong $\mathrm{Ta}^{2}$ \\ HCMC University of Technology and Education \\ Ho Chi Minh City, Vietnam
}

\begin{abstract}
This research proposes a $\mathbf{H}^{\infty}$ robust recurrent cerebellar model articulation control system (RRCMACS) for MIMO non-linear systems to achieve the robustness of the system during operation. In this system, the superior properties of the recurrent cerebellar model articulation controller (RCMAC) are incorporated to imitate an ideal sliding mode controller. The $\mathrm{H}^{\infty}$ robust controller efficiently attenuates the effects of uncertainties, external disturbances, and noises to maintain the robustness of the system. The parameters of the controller were updated in the sense of the Lyapunov-like Lemma theory. Therefore, the stability and robustness of the system were guaranteed. The simulation results for the micromotion stage system are given to prove the effectiveness and applicability of the proposed control system for model-free nonlinear systems.
\end{abstract}

Keywords-Robust controller; MIMO non-linear systems; linear piezoelectric motor (LPM); recurrent network; Cerebellar model articulation controller

\section{INTRODUCTION}

Most of the practical applications are non-linear systems. The reason for this comes from the effects of friction and damping coefficients, cross-coupling, hysteresis phenomenon, and time-varying parameters [1-4]. The completely dynamic model of the practical systems can't be obtained. Therefore, the model-based controllers cannot achieve good performance for the non-linear MIMO systems [5-6].

To cope with the drawbacks of the model-based controllers, many modern controllers have been developed such as Fuzzy Logic Controller (FLC), Sliding Mode Controller (SMC), Neural Networks (NNs), and Cerebellar Model Articulation Controller (CMAC). In particular, the FLC was considered an effective control method for the uncertainties, free-model and non-linear systems. The essential property of the FLC is that the non-linear and uncertain parts are described as fuzzy sets and rules [7-9]. Consequently, the FLC overcomes the shortcomings of the model-based controllers in dealing with the non-linear, uncertainties systems. However, the performance of the FLC depends utterly on the selection of fuzzy sets and the number of rules. There are not specific methods that ensure the optimal selections of fuzzy sets and rules for the controllers so far. To achieve good performance for the practical applications, the fuzzy sets and rules were mostly selected by trial and error.
To deal with the effects of uncertainties, disturbances, and noises in non-linear systems, the SMC was developed and applied [10-11]. The SMC guarantees response of the system becomes insensitive to disturbances and noise in the sliding phase. Consequently, the robustness of the system can be guaranteed. The disadvantages and limitations of the SMC are the chattering phenomena and the selection of the boundary of uncertainties or disturbances. These problems are serious difficulty and impossible tasks in realistic applications.

Along with the SMC, the neural network (NN) was used to approximate non-linear functions to arbitrary precision. Therefore, it has been proposed for dealing with non-linear systems and obtained good results in realistic applications [1213]. The NNs, however, have shortcomings that attracted much attention from the researchers so far. In particular, all weights in the structure of the neural network are updated each learning cycle, this is unsuitable for the problems requiring real-time learning; the selection of the number of neurons and hidden layers to achieve good performances is very difficult to obtain in the practical applications.

In recent decades, the Cerebellar Model Articulation Controller (CMAC) has been developed and adopted for the complex non-linear MIMO systems due to it has superior properties to NNs [14-17]. To improve learning capability and dynamic response of the CMAC, the wavelet function and recurrent technique were incorporated into the CMAC to improve the performance of the system [18].

Although the above researches achieved good results in designing the controllers to cope with the high non-linear MIMO systems, the robustness of the system in the presence of disturbances and sensor noise were not totally mentioned.

In this research, a $\mathrm{H}^{\infty}$ robust recurrent cerebellar model articulation control system (RRCMACS) is proposed for the non-linear MIMO system. Therein, the RCMAC is used to imitate the ideal sliding mode controller to minimize error surface and the $\mathrm{H}^{\infty}$ robust controller is utilized to attenuate the effects of disturbances, uncertainties, and sensor noise acting on the system to achieve the $\mathrm{H}^{\infty}$ robustness performance for the overall system.

The paper is organized as follows: Section 2 presents nonlinear system and proposed control system. Section 3 describes the structure of the RCMAC and the $\mathrm{H}^{\infty}$ robust controller. The simulation results are provided in Section 4. Section 5 presents conclusions and future works. 


\section{Problem Formulation AND PROPOSED CONTROL SYSTEM}

In general, the dynamic equation of MIMO non-linear systems including disturbances, uncertainties, and noise is described below:

$\left\{\begin{array}{l}\mathbf{x}^{\mathrm{n}}=\mathbf{F}_{0}(\underline{\mathbf{x}})+\Delta \mathbf{F}(\underline{\mathbf{x}})+\left(\mathbf{G}_{0}(\underline{\mathbf{x}})+\Delta \mathbf{G}(\underline{\mathbf{x}})\right) \mathbf{u}+\mathbf{d n}(\underline{\mathbf{x}}) \\ \quad=\mathbf{F}_{\mathbf{0}}(\underline{\mathbf{x}})+\mathbf{G}_{\mathbf{0}}(\underline{\mathbf{x}}) \mathbf{u}+\mathbf{U D}(\underline{\mathbf{x}}) \\ \mathbf{y}=\mathbf{x}\end{array}\right.$

where $\mathbf{y}=\mathbf{x}=\left[\mathrm{x}_{1}, \mathrm{x}_{2}, \ldots, \mathrm{x}_{\mathrm{n}_{0}}\right]^{\mathrm{T}} \in \mathrm{R}^{\mathrm{n}_{0}}$ is the system output vector, $\underline{\mathbf{x}}=\left[\mathrm{x}^{\mathrm{T}}, \dot{\mathrm{x}}^{\mathrm{T}}, \ldots, \mathrm{x}^{(\mathrm{n}-1) \mathrm{T}}\right]^{\mathrm{T}} \in \mathrm{R}^{\mathrm{n}}$ is the system state vector, $\mathbf{u}=\left[\mathrm{u}_{1}, \mathrm{u}_{2}, \ldots, \mathrm{u}_{\mathrm{n}_{0}}\right]^{\mathrm{T}} \in \mathrm{R}^{\mathrm{n}_{0}}$ is the control input vector, $\mathbf{F}_{0}(\underline{\mathbf{x}}) \in \mathrm{R}^{\mathrm{n}_{0} \times \mathrm{n}_{0}} \quad$ in the nominal non-linear function, $\mathbf{G}_{0}(\underline{\mathbf{x}}) \in \mathrm{R}^{\mathrm{n}_{0} \times \mathrm{n}_{0}}$ is the nominal gain matrix, $\Delta \mathbf{F}(\underline{\mathbf{x}})$ and $\Delta \mathbf{G}(\underline{\mathbf{x}})$ are the changes in parameters of the $\mathbf{F}_{0}(\underline{\mathbf{x}}) \in \mathrm{R}^{\mathrm{n}_{0} \times \mathrm{n}}$, and $\quad \mathbf{G}_{0}(\underline{\mathbf{x}}) \in \mathrm{R}^{\mathrm{n}_{0} \times \mathrm{n}_{0}}, \quad$ respectively. $\mathbf{d n}(\underline{\mathbf{x}})=\left[\mathrm{dn}_{1}, \mathrm{dn}_{2}, \ldots, \mathrm{dn}_{0}\right]^{\mathrm{T}} \in \mathrm{R}^{\mathrm{n}_{0}}$ stands for external disturbances and noise $\quad \mathbf{U D}(\underline{\mathbf{x}})=\Delta \mathbf{F}(\underline{\mathbf{x}})+\Delta \mathbf{G}(\underline{\mathbf{x}}) \mathbf{u}+\mathbf{d n}(\underline{\mathbf{x}})$ is lumped uncertainties, disturbances, and noise. The objective of the control system is designed so that the output signals $\mathbf{x}$ can only track desired trajectories $\mathbf{x}_{\mathrm{d}} \in \mathrm{R}^{\mathrm{n}_{\mathrm{o}}}$ but also satisfy robust performance in the presence of the uncertainties, disturbances, and noise

For the high-order system, the sliding error manifold is defined [18] to reduce the order of variables during designing and computation the control system, the sliding error manifold has the following form:

$$
\mathbf{S}=\mathbf{e}^{\mathrm{n}-1}+\mathbf{K} \underline{\mathbf{e}}
$$

Therein, $\mathbf{e}=\mathbf{x}_{\mathrm{d}}-\mathbf{x}$ and $\underline{\mathbf{e}}=\left[\mathbf{e}, \dot{\mathbf{e}}, \ldots, \mathbf{e}^{\mathbf{n}-\mathbf{1}}\right]^{\mathrm{T}}$ are the tracking error and error vector of the system, respectively. Derivative both sides of $\mathbf{S}$ and combination with the dynamic equation (1), yields.

$$
\begin{aligned}
& \dot{\mathrm{S}}=\mathrm{e}^{\mathrm{n}}+\mathrm{K} \underline{\dot{\mathrm{e}}}=\mathrm{x}_{d}^{\mathrm{n}}-\mathrm{x}^{\mathrm{n}}+\mathrm{K}(\underline{\dot{\mathrm{e}}}) \\
& =x_{d}^{n}-F_{0}(\underline{x})-G_{0}(\underline{x}) \mathbf{u}-U D(\underline{x})+K(\underline{\dot{e}})
\end{aligned}
$$

In case of the nominal values $\mathbf{F}_{0}(\underline{\mathbf{x}}) \in \mathrm{R}^{\mathrm{n}_{0} \mathrm{x}_{0}}$, $\mathbf{G}_{0}^{-1}(\underline{\mathbf{x}}) \in \mathrm{R}^{\mathrm{n}_{0} \mathrm{xn}_{0}}$, the lumped of external disturbances, uncertainties, and noise $\mathbf{U D}(\underline{\mathbf{x}})$ are exactly known, an ideal sliding mode (ISM) controller is designed to guarantee the stability of the system as follows [19]:

$$
\mathbf{u}_{\mathrm{ISM}}=\mathbf{G}_{0}^{-1}(\underline{\mathbf{x}})\left[\mathbf{x}_{\mathbf{d}}^{\mathrm{n}}-\mathbf{F}_{0}(\underline{\mathbf{x}})-\mathbf{U D}(\underline{\mathbf{x}})+\mathbf{K}(\underline{\dot{e}})+\boldsymbol{\eta} \operatorname{sgn}(\mathbf{S})\right]
$$

Proof: According to the sliding mode control [9-10], the Lyapunov function candidate is selected to prove the stability of the system as follows:

$$
\mathbf{V}(\mathbf{t})=\frac{1}{2} \mathbf{S}^{2}
$$

Derivative two sides of Lyapunov function candidate, and replacing $\mathbf{u}$ in (5) by $\mathbf{u}_{\text {ISM }}$ in (4), yields

$$
\dot{\mathbf{V}}(\mathbf{t})=\mathbf{S} \dot{\mathbf{S}}=-\mathbf{S} \boldsymbol{\eta} \operatorname{sgn}(\mathbf{S}) \leq-\boldsymbol{\eta}|\mathbf{S}|
$$

The stability of the system is guaranteed in case of any $\boldsymbol{\eta}>\mathbf{0}$

However, for the complex high non-linear systems, the external disturbances, uncertainties, and noise $\mathbf{U D}(\underline{\mathbf{x}})$ cannot be defined, measured or estimated exactly in practical applications. Consequently, the $\mathbf{u}_{\text {ISM }}$ cannot satisfy the stability and robust performance of the system. To handle these problems, a $\mathrm{H}^{\infty}$ robust recurrent cerebellar model articulation control system (RRCMACS) is proposed and depicted in Fig. 1. In this system, superior properties of the recurrent cerebellar model articulation controller (RCMAC) are incorporated to imitate the ideal sliding mode controller and the $\mathrm{H}^{\infty}$ robust controller efficiently attenuates the effects of external disturbances, noise, and uncertainties to a prescribed level to maintain the robustness of the system. The proposed control system has the following form:

$\mathbf{u}_{\mathrm{RRCMACS}}=\mathbf{u}_{\mathrm{ISM}}-\mathbf{u}_{\mathrm{RC}}-\mathbf{u}_{\mathrm{RCMAC}}$

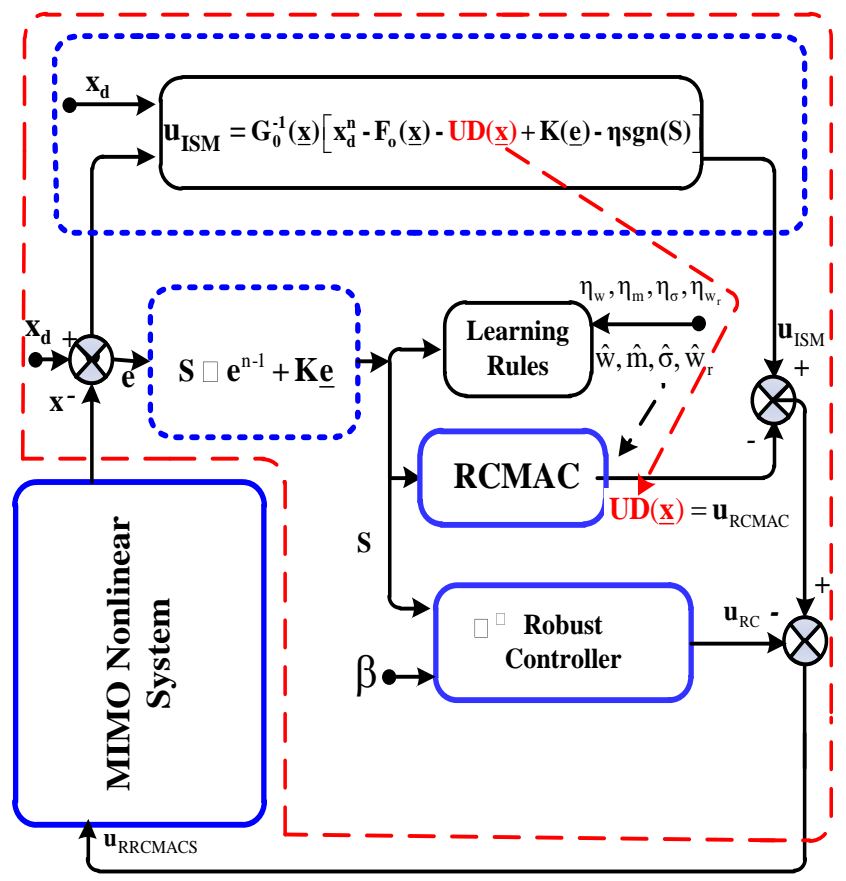

Fig. 1. Structure of the Proposed Control System. 


\section{THE RRCMAC SYSTEM DESIGN}

\section{A. The RCMAC}

The $\mathbf{u}_{\text {RCMAC }}$ with its fast learning, good generalization, and dynamic response capability of the CMACs [18] were utilized to mimic the ideal sliding mode controller, $\mathbf{u}_{\text {ISM }}$ in the presence of the uncertainties, disturbance, noise to minimize the error sliding manifold, $\mathbf{S}$.

The structure of the RCMAC is depicted in Fig. 2 including input Space $\mathbf{S}$, association memory space $\mathbf{A}$, receptive field space $\mathbf{R}$, weight memory space $\mathbf{W}$, and output spaces $\mathbf{O}$.

The signal propagation in the RCMAC is described as follows:

$\mathrm{S}_{\mathrm{ri}}(\mathrm{k})=\mathrm{S}_{\mathrm{i}}(\mathrm{k})+\mathrm{w}_{\text {rik }} \mu_{\mathrm{ik}}(\mathrm{k}-1), \mathrm{i}=1,2, \ldots, \mathrm{n}$

$\mu_{\mathrm{ik}}\left(\mathrm{S}_{\mathrm{ri}}\right)=\exp \left[-\frac{\left(\mathrm{S}_{\mathrm{ri}}-\mathrm{m}_{\mathrm{ik}}\right)^{2}}{\sigma_{\mathrm{ik}}^{2}}\right], \mathrm{k}=1,2, \ldots, \mathrm{n}_{\mathrm{b}}$

$b_{i k}=\prod_{i=1}^{n} \mu_{i k}\left(S_{r i}\right), i=1,2, \ldots, n, k=1,2, \ldots, n_{b}$

$\mathrm{O}_{\mathrm{j}}=\sum_{\mathrm{j}=1}^{\mathrm{n}_{0}} \sum_{\mathrm{k}=1}^{\mathrm{n}_{\mathrm{b}}} \mathrm{w}_{\mathrm{jk}} \prod_{\mathrm{i}=1}^{\mathrm{n}} \mu_{\mathrm{ik}}\left(\mathrm{S}_{\mathrm{ri}}\right)=\mathrm{w}^{\mathrm{T}} \mathbf{b}, \mathrm{j}=1,2, \ldots, \mathrm{n}_{0}$

Where $\mathrm{n}, \mathrm{n}_{\mathrm{o}}$, and $\mathrm{n}_{\mathrm{b}}$ are the number of inputs, outputs, and blocks in receptive space; $\mathrm{S}_{\mathrm{r}}, \mu, \mathrm{b}$, and $\mathrm{O}$ are the input data including recurrent elements, Gaussian function, overlapped receptive space, and output data, respectively; $\mathrm{m}$ and $\sigma$ are mean and deviation of Gaussian function; $w_{r}$ and $w$ are recurrent and output weights.

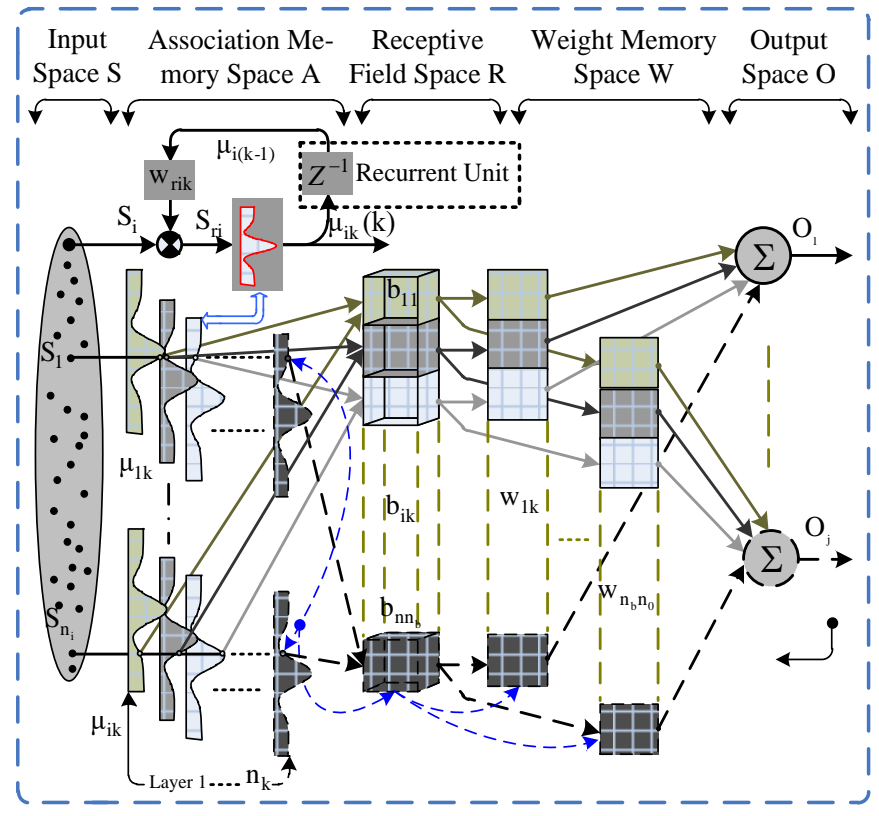

Fig. 2. The Structure of the RCMAC.

\section{B. The Robust Controller Design}

The RCMAC aims to imitate $\mathbf{u}_{\mathrm{ISM}}$ in (4). In the case of ideal designing, meanwhile, the parameters of the system are exactly known, external disturbances and uncertainties are absent. An ideal approximation controller can be described as follows:

$\mathbf{u}_{\mathrm{RCMAC}}^{0}\left(\mathbf{S}^{0}, \mathbf{m}^{0}, \boldsymbol{\sigma}^{0}, \mathbf{w}_{\mathbf{r}}^{0}, \mathbf{w}^{0}\right)=\mathbf{w}^{0 \mathrm{~T}} \mathbf{b}^{0}+\boldsymbol{\varepsilon}$

However, defining exactly the nominal parameters of the system is unobtainable in practical applications. Furthermore, the disturbances and noise always exist in the non-linear systems. Consequently, the estimated parameters are used instead of nominal parameters in designing the $\mathbf{u}_{\text {RRCMACS }}$ as follows:

$\mathbf{u}_{\mathrm{RRCMACS}}\left(\hat{\mathbf{S}}, \hat{\mathbf{m}}, \hat{\boldsymbol{\sigma}}, \hat{\mathbf{w}}_{\mathbf{r}}, \hat{\mathbf{w}}\right)+\mathbf{u}_{\mathrm{RC}}=\hat{\mathrm{w}}^{\mathrm{T}} \hat{\mathbf{b}}+\mathbf{u}_{\mathrm{RC}}$

Therein, $\hat{\mathbf{S}}, \hat{\mathbf{m}}, \hat{\boldsymbol{\sigma}}, \hat{\mathbf{w}}_{\mathbf{r}}, \hat{\mathbf{w}}$ is an estimation of the nominal parameters $\mathbf{S}^{0}, \mathbf{m}^{0}, \boldsymbol{\sigma}^{0}, \mathbf{w}_{\mathbf{r}}^{0}, \mathbf{w}^{0}$ and $\mathbf{u}_{\mathrm{RC}}$ is the robust controller which attenuates the effects of the uncertainties, disturbances, and noise to guarantee the robustness of the system. Comprising (4) and (3), the error dynamic is rewritten as follows:

$\dot{\mathbf{S}}=\mathbf{G}_{0}(\underline{\mathbf{x}})\left(\mathbf{u}_{\mathrm{ISM}}-\mathbf{u}\right)-\boldsymbol{\eta} \operatorname{sgn}(\mathbf{S})$

By replacing (12) and (13) into (14), yields

$$
\begin{aligned}
\dot{\mathbf{S}} & =\mathbf{G}_{0}(\underline{\mathbf{x}})\left(\mathbf{u}_{\mathrm{RCMAC}}^{\mathbf{0}}-\mathbf{u}_{\mathrm{RRCMACS}}\right)-\boldsymbol{\eta} \operatorname{sgn}(\mathbf{S}) \\
& =\mathbf{G}_{0}(\underline{\mathbf{x}})\left(\mathbf{w}^{0 \mathrm{~T}} \mathbf{b}^{\mathbf{0}}-\hat{\mathbf{w}}^{\mathrm{T}} \hat{\mathbf{b}}+\boldsymbol{\varepsilon}-\mathbf{u}_{\mathrm{RC}}\right)-\boldsymbol{\eta} \operatorname{sgn}(\mathbf{S}) \\
& =\mathbf{G}_{0}(\underline{\mathbf{x}})\left(\tilde{\mathbf{w}}^{\mathrm{T}} \mathbf{b}^{\mathbf{0}}+\hat{\mathbf{w}}^{\mathrm{T}} \tilde{\mathbf{b}}+\boldsymbol{\varepsilon}-\mathbf{u}_{\mathrm{RC}}\right)-\boldsymbol{\eta} \operatorname{sgn}(\mathbf{S})
\end{aligned}
$$

Where $\tilde{\mathbf{w}}=\mathbf{w}^{\mathbf{0}}-\hat{\mathbf{w}}, \tilde{\mathbf{b}}=\mathbf{b}^{\mathbf{0}}-\hat{\mathbf{b}}$ is an error of estimation.

According to the linearization technique, Taylor series expansion is used to transform the multi-dimension receptivefield space into a partially linear form. A linear approximation of $\tilde{\mathbf{b}}$ in three variables $\mathbf{m}, \boldsymbol{\sigma}$ and $\mathbf{w}_{\mathrm{r}}$ has the following form:

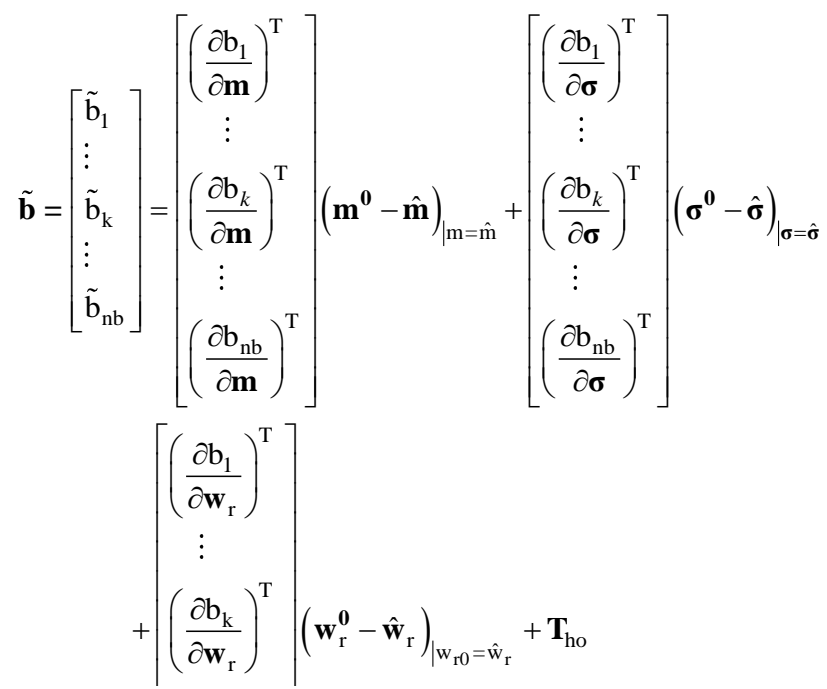




$$
\begin{aligned}
& \left\lfloor\left(\begin{array}{c}
\vdots \\
\left.\frac{\partial \mathrm{b}_{\mathrm{nb}}}{\partial \mathbf{w}_{\mathrm{r}}}\right)^{\mathrm{T}}
\end{array}\right\rfloor\right. \\
& =\mathbf{C}^{\mathrm{T}} \tilde{\mathbf{m}}+\mathbf{E}^{\mathrm{T}} \tilde{\boldsymbol{\sigma}}+\mathbf{F}^{\mathrm{T}} \tilde{\mathbf{w}}_{\mathrm{r}}+\mathbf{T}_{\mathrm{ho}}
\end{aligned}
$$

where $\quad \tilde{\mathbf{b}}=\mathbf{b}^{0}-\hat{\mathbf{b}}, \tilde{\boldsymbol{\sigma}}=\boldsymbol{\sigma}^{0}-\hat{\boldsymbol{\sigma}}, \tilde{\mathbf{w}}=\mathbf{w}_{\mathrm{r}}^{\mathbf{0}}-\hat{\mathbf{w}}_{\mathrm{r}}$, and $\quad \mathbf{T}_{\mathrm{ho}}$ is higher-order terms of Taylor series expansion.

$$
\begin{aligned}
& \mathbf{C}^{\mathrm{T}}=\left[\frac{\partial \mathrm{b}_{1}}{\partial \mathbf{m}}, \cdots, \frac{\partial \mathrm{b}_{\mathrm{k}}}{\partial \mathbf{m}}, \cdots \frac{\partial \mathrm{b}_{\mathrm{nb}}}{\partial \mathbf{m}}\right]_{\mid \mathbf{m}=\hat{\mathbf{m}}} \in \mathfrak{R}^{\mathrm{n}_{\mathrm{i}} \mathrm{n}_{\mathrm{b}} \times \mathrm{n}_{\mathrm{b}}} \\
& \mathbf{E}^{\mathrm{T}}=\left[\frac{\partial \mathrm{b}_{1}}{\partial \boldsymbol{\sigma}}, \cdots, \frac{\partial \mathrm{b}_{\mathrm{k}}}{\partial \boldsymbol{\sigma}}, \cdots \frac{\partial \mathrm{b}_{\mathrm{nb}}}{\partial \boldsymbol{\sigma}}\right]_{\mid \boldsymbol{\sigma}=\hat{\boldsymbol{\sigma}}} \in \mathfrak{R}^{\mathrm{n}_{\mathrm{n}} \mathrm{n}_{\mathrm{b}} \times \mathrm{n}_{\mathrm{b}}} \\
& \mathbf{F}^{\mathrm{T}}=\left[\frac{\partial \mathrm{b}_{1}}{\partial \mathbf{w}_{\mathrm{r}}}, \cdots, \frac{\partial \mathrm{b}_{\mathrm{k}}}{\partial \mathbf{w}_{\mathrm{r}}}, \cdots \frac{\partial \mathrm{b}_{\mathrm{nb}}}{\partial \mathbf{w}_{\mathrm{r}}}\right]_{\left.\right|_{\mathbf{w}_{\mathrm{r}}=\hat{\mathbf{w}}_{\mathrm{r}}} \in \mathfrak{R}^{\mathrm{n}_{\mathrm{i}} \mathrm{n}_{\mathrm{b}} \times \mathrm{n}_{\mathrm{b}}}}
\end{aligned}
$$

Substitution $\mathbf{C}^{\mathrm{T}} \tilde{\mathbf{m}}+\mathbf{E}^{\mathrm{T}} \tilde{\boldsymbol{\sigma}}+\mathbf{F}^{\mathrm{T}} \tilde{\mathbf{w}}_{\mathrm{r}}+\mathbf{T}_{\mathrm{ho}}$ and $\mathbf{b}^{0}=\hat{\mathbf{b}}+\tilde{\mathbf{b}}$ into error dynamic equation (15), yields

$$
\begin{aligned}
& \dot{\mathbf{S}}=\mathbf{G}_{0}(\underline{\mathbf{x}})\left(\tilde{\mathbf{w}}^{\mathrm{T}} \mathbf{b}^{\mathbf{0}}+\hat{\mathbf{w}}^{\mathrm{T}} \tilde{\mathbf{b}}+\boldsymbol{\varepsilon}-\mathbf{u}_{\mathrm{RC}}\right)-\boldsymbol{\eta} \operatorname{sgn}(\mathbf{S}) \\
& =\mathbf{G}_{0}(\underline{\mathbf{x}})\left(\tilde{\mathbf{w}}^{\mathrm{T}}\left(\hat{\mathbf{b}}+\mathbf{C}^{\mathrm{T}} \tilde{\mathbf{m}}+\mathbf{E}^{\mathrm{T}} \tilde{\boldsymbol{\sigma}}+\mathbf{F}^{\mathrm{T}} \tilde{\mathbf{w}}_{\mathrm{r}}+\mathbf{T}_{\mathrm{ho}}\right)\right. \\
& \left.+\hat{\mathbf{w}}^{\mathrm{T}}\left(\mathbf{C}^{\mathrm{T}} \tilde{\mathbf{m}}+\mathbf{E}^{\mathrm{T}} \tilde{\boldsymbol{\sigma}}+\mathbf{F}^{\mathrm{T}} \tilde{\mathbf{w}}_{\mathrm{r}}+\mathbf{T}_{\mathrm{ho}}\right)+\boldsymbol{\varepsilon}-\mathbf{u}_{\mathrm{RC}}\right)-\boldsymbol{\eta} \operatorname{sgn}(\mathbf{s}) \\
& =\mathbf{G}_{0}(\underline{\mathbf{x}})\left(\tilde{\mathbf{w}}^{\mathrm{T}} \hat{\mathbf{b}}+\hat{\mathbf{w}}^{\mathrm{T}}\left(\mathbf{C}^{\mathrm{T}} \tilde{\mathbf{m}}+\mathbf{E}^{\mathrm{T}} \tilde{\boldsymbol{\sigma}}+\mathbf{F}^{\mathrm{T}} \tilde{\mathbf{w}}_{\mathrm{r}}\right)\right. \\
& \left.+\tilde{\mathbf{w}}^{\mathrm{T}}\left(\mathbf{C}^{\mathrm{T}} \tilde{\mathbf{m}}+\mathbf{E}^{\mathrm{T}} \tilde{\boldsymbol{\sigma}}+\mathbf{F}^{\mathrm{T}} \tilde{\mathbf{w}}_{\mathrm{r}}\right)+\mathbf{w}^{0 \mathrm{~T}} \mathbf{T}_{\mathrm{ho}}+\boldsymbol{\varepsilon}-\mathbf{u}_{\mathrm{RC}}\right)-\boldsymbol{\eta} \operatorname{sgn}(\mathbf{S}) \\
& =\mathbf{G}_{0}(\underline{\mathbf{x}})\left(\tilde{\mathbf{w}}^{\mathrm{T}} \hat{\mathbf{b}}+\hat{\mathbf{w}}^{\mathrm{T}}\left(\mathbf{C}^{\mathrm{T}} \tilde{\mathbf{m}}+\mathbf{E}^{\mathrm{T}} \tilde{\boldsymbol{\sigma}}+\mathbf{F}^{\mathrm{T}} \tilde{\mathbf{w}}_{\mathrm{r}}\right)-\mathbf{u}_{\mathrm{RC}}\right.
\end{aligned}
$$

$+\mathbf{U D}(\underline{\mathbf{x}}))-\eta \operatorname{sgn}(\mathbf{S})$

where $\quad \mathbf{U D}(\underline{\mathbf{x}})=\tilde{\mathbf{w}}^{\mathrm{T}}\left(\mathbf{C}^{\mathrm{T}} \tilde{\mathbf{m}}+\mathbf{E}^{\mathrm{T}} \tilde{\boldsymbol{\sigma}}+\mathbf{F}^{\mathrm{T}} \tilde{\mathbf{w}}_{\mathrm{r}}\right)+\mathbf{w}^{0 \mathrm{~T}} \mathbf{T}_{\mathrm{ho}}+\boldsymbol{\varepsilon}$ stands for the external disturbances, noises, and uncertainties

In other to prove the stability and robustness of the system under the effects of disturbances, noises, and uncertainties, the Lyapunov function is chosen as follows:

$\mathbf{V}\left(\mathbf{S}, \tilde{\mathbf{w}}, \tilde{\mathbf{m}}, \tilde{\boldsymbol{\sigma}}, \tilde{\mathbf{w}}_{\mathrm{r}}\right)=\frac{1}{2} \mathbf{S}^{\mathrm{T}} \mathbf{G}_{0}^{-1}(\underline{\mathbf{x}}) \mathbf{S}+\frac{1}{2 \eta_{\mathrm{w}}} \tilde{\mathbf{w}}^{\mathrm{T}} \tilde{\mathbf{w}}$

$+\frac{1}{2 \eta_{\mathrm{m}}} \tilde{\mathbf{m}}^{\mathrm{T}} \tilde{\mathbf{m}}+\frac{1}{2 \eta_{\sigma}} \tilde{\boldsymbol{\sigma}}^{\mathrm{T}} \tilde{\boldsymbol{\sigma}}+\frac{1}{2 \eta_{\mathrm{w}_{\mathrm{r}}}} \tilde{\mathbf{w}}_{\mathrm{r}}^{\mathrm{T}} \tilde{\mathbf{w}}_{\mathrm{r}}$

By selection the adaptive rules as (22)-(25), the $\mathrm{H}^{\infty}$ robustness performance of the system are satisfied.

$$
\begin{aligned}
& \dot{\hat{\mathbf{w}}}=\frac{1}{\eta_{\mathrm{w}}} \hat{\mathbf{b}} \mathbf{S}^{\mathrm{T}} \\
& \dot{\hat{\mathbf{m}}}=\frac{1}{\eta_{\mathrm{m}}} \mathbf{C}^{\mathrm{T}} \hat{\mathbf{w}} \mathbf{S}
\end{aligned}
$$

$$
\begin{aligned}
& \dot{\hat{\boldsymbol{\sigma}}}=\frac{1}{\eta_{\sigma}} \mathbf{E}^{\mathrm{T}} \hat{\mathbf{w}} \mathbf{S} \\
& \dot{\hat{\mathbf{w}}}_{\mathrm{r}}=\frac{1}{\eta_{\mathrm{w}_{\mathrm{r}}}} \mathbf{F}^{\mathrm{T}} \hat{\mathbf{w}} \mathbf{S}
\end{aligned}
$$

Proof: Taking derivative two sides of the Lyapunov function (21), yields

$$
\begin{gathered}
\left.\dot{\mathbf{V}}=\mathbf{S}^{\mathrm{T}} \mathbf{G}_{0}^{-1}(\underline{\mathbf{x}}) \dot{\mathbf{S}}+\eta_{\mathrm{w}} \tilde{\mathbf{w}}^{\mathrm{T}} \dot{\tilde{\mathbf{w}}}+\eta_{\mathrm{m}} \tilde{\mathbf{m}}^{\mathrm{T}} \dot{\tilde{\mathbf{m}}}+\eta_{\sigma} \tilde{\boldsymbol{\sigma}}^{\mathrm{T}} \dot{\tilde{\boldsymbol{\sigma}}}+\eta_{\mathrm{w}_{\mathrm{r}}} \tilde{\mathbf{w}}_{\mathrm{r}}^{\mathrm{T}} \dot{\tilde{\mathbf{w}}}_{\mathrm{r}}\right) \\
=\mathbf{S}^{\mathrm{T}}\left(\tilde{\mathbf{w}}^{\mathrm{T}} \hat{\mathbf{b}}+\hat{\mathbf{w}}^{\mathrm{T}}\left(\mathbf{C}^{\mathrm{T}} \tilde{\mathbf{m}}+\mathbf{E}^{\mathrm{T}} \tilde{\boldsymbol{\sigma}}+\mathbf{F}^{\mathrm{T}} \tilde{\mathbf{w}}_{\mathrm{r}}\right)-\mathbf{u}_{\mathrm{RC}}+\mathbf{U D}(\underline{\mathbf{x}})\right) \\
-\mathbf{S}^{\mathrm{T}} \mathbf{G}_{0}^{-1} \boldsymbol{\eta} \operatorname{sgn}(\mathbf{S})-\eta_{\mathrm{w}} \tilde{\mathbf{w}}^{\mathrm{T}} \dot{\hat{\mathbf{w}}}-\eta_{\mathrm{m}} \tilde{\mathbf{m}}^{\mathrm{T}} \dot{\hat{\mathbf{m}}}-\eta_{\sigma} \tilde{\boldsymbol{\sigma}}^{\mathrm{T}} \dot{\hat{\boldsymbol{\sigma}}}-\eta_{\mathrm{w}_{\mathrm{r}}} \tilde{\mathbf{w}}_{\mathrm{r}}^{\mathrm{T}} \dot{\tilde{\mathbf{w}}}_{\mathrm{r}}
\end{gathered}
$$

Due to $\mathbf{S}^{\mathrm{T}} \tilde{\mathbf{w}}^{\mathrm{T}} \hat{\mathbf{b}}=\tilde{\mathbf{w}}^{\mathrm{T}} \hat{\mathbf{b}} \mathbf{S}^{\mathrm{T}}$ and $\mathbf{S}^{\mathrm{T}} \mathbf{G}_{0}^{-1} \boldsymbol{\eta} \operatorname{sgn}(\mathbf{S}) \geq \mathbf{0}$, therefore, the equation (26) can be rewritten as below:

$$
\begin{aligned}
& \dot{\mathbf{V}} \leq \tilde{\mathbf{w}}^{\mathrm{T}}\left(\hat{\mathbf{b}} \mathbf{S}^{\mathrm{T}}-\eta_{\mathrm{w}} \dot{\hat{\mathbf{w}}}\right)+\tilde{\mathbf{m}}^{\mathrm{T}}\left(\mathbf{C}^{\mathrm{T}} \hat{\mathbf{w}} \mathbf{S}-\eta_{\mathrm{m}} \dot{\hat{\mathbf{m}}}\right) \\
& +\tilde{\boldsymbol{\sigma}}^{\mathrm{T}}\left(\mathbf{E}^{\mathrm{T}} \hat{\mathbf{w}} \mathbf{S}-\eta_{\sigma} \dot{\hat{\boldsymbol{\sigma}}}\right)+\tilde{\mathbf{w}}_{\mathrm{r}}^{\mathrm{T}}\left(\mathbf{F}^{\mathrm{T}} \hat{\mathbf{w}} \mathbf{S}-\eta_{\mathrm{w}_{\mathrm{r}}} \dot{\hat{\mathbf{w}}}_{\mathrm{r}}\right) \\
& +\mathbf{S}^{\mathrm{T}} \mathbf{U D}(\underline{\mathbf{x}})-\mathbf{S}^{\mathrm{T}} \mathbf{u}_{\mathrm{RC}}
\end{aligned}
$$
yields

By replacing the adaptive rules from (22)-(25) into (27),

$\dot{\mathbf{V}}\left(\mathbf{S}, \tilde{\mathbf{w}}, \tilde{\mathbf{m}}, \tilde{\boldsymbol{\sigma}}, \tilde{\mathbf{w}}_{\mathrm{r}}\right) \leq \mathbf{S}^{\mathrm{T}} \mathbf{U D}(\underline{\mathbf{x}})-\mathbf{S}^{\mathrm{T}} \mathbf{u}_{\mathrm{RC}}$

$$
=\sum_{\mathrm{i}=1}^{\mathrm{n}}\left(\mathrm{S}_{\mathrm{i}} \mathrm{UD}_{\mathrm{i}}(\underline{\mathrm{x}})-\mathrm{S}_{\mathrm{i}} \mathbf{u}_{\mathrm{RC}}\right)
$$

Selecting control law for the robust controller as follows:

$\mathbf{u}_{\mathrm{RC}}=\frac{\left(\beta_{\mathrm{i}}^{2}+1\right) \mathrm{S}_{\mathrm{i}}}{2 \beta_{\mathrm{i}}^{2}}$

By replacing the $\mathbf{u}_{\mathrm{RC}}$ into (28), yields

Integrating two sides of equation (30) from $t=0$ to $t=\infty$, yields $\mathrm{t}=0$

$$
\begin{aligned}
& \dot{\mathbf{v}} \leq=\sum_{i=1}^{n}\left(S_{i} U D_{i}(\underline{x})-S_{i} \frac{\left(\beta_{i}^{2}+1\right) S_{i}}{2 \beta_{i}^{2}}\right) \\
& \leq \sum_{i=1}^{n}\left(S_{i} U D_{i}(\underline{x})-\frac{1}{2} S_{i}^{2}-\frac{1}{2 \beta_{i}^{2}} S_{i}^{2}\right. \\
& \leq \sum_{i=1}^{n}\left(-\frac{1}{2} S_{i}^{2}-\frac{1}{2}\left(\frac{S_{i}}{\beta_{i}}-\beta_{i} U D_{i}(\underline{x})\right)^{2}\right. \\
& +\frac{\beta_{i}^{2} U D_{i}(\underline{x})^{2}}{2} \\
& \leq \sum_{i=1}^{n}\left(-\frac{1}{2} S_{i}^{2}+\frac{\beta_{i}^{2} U D_{i}(\underline{x})^{2}}{2}\right)
\end{aligned}
$$


$V(T)-V(0) \leq \sum_{i=1}^{n}\left(-\frac{1}{2} \int_{t=0}^{t=\infty} S_{i}^{2} d t\right.$

$\left.+\frac{\beta_{\mathrm{i}}^{2}}{2} \int_{\mathrm{t}=0}^{\mathrm{t}=\infty} \mathrm{UD}_{\mathrm{i}}(\underline{\mathrm{x}})^{2} \mathrm{dt}\right)$

Because of the value of the Lyapunov function, $\mathrm{V}(\mathrm{T}) \geq 0$, the inequality in (31) can be described as follows:

$$
\begin{array}{r}
\mathrm{V}(\mathrm{T})+\sum_{\mathrm{i}=1}^{\mathrm{n}}\left(\frac{1}{2} \int_{\mathrm{t}=0}^{\mathrm{T}} \mathrm{S}_{\mathrm{i}}^{2} \mathrm{dt} \leq \mathrm{V}(0)+\sum_{\mathrm{i}=0}^{\mathrm{i}=\mathrm{n}} \frac{\beta_{\mathrm{i}}^{2}}{2} \int_{\mathrm{t}=0}^{\mathrm{T}} \mathrm{UD}_{\mathrm{i}}(\underline{\mathrm{x}})^{2} \mathrm{dt}\right. \\
\sum_{\mathrm{i}=1}^{\mathrm{n}} \frac{1}{2} \int_{\mathrm{t}=0}^{\mathrm{T}} \mathrm{S}_{\mathrm{i}}^{2} \mathrm{dt} \leq \mathrm{V}(0)+\sum_{\mathrm{i}=0}^{\mathrm{i}=\mathrm{n}} \frac{\beta_{\mathrm{i}}^{2}}{2} \int_{\mathrm{t}=0}^{\mathrm{T}} \mathrm{UD}_{\mathrm{i}}(\underline{\mathrm{x}})^{2} \mathrm{dt}
\end{array}
$$

Basing on the equation of the Lyapunov function in (21), the inequality in (32) is equivalent to the following form:

$$
\begin{aligned}
& \sum_{\mathrm{i}=1}^{\mathrm{n}}\left(\frac{1}{2} \int_{\mathrm{t}=0}^{\mathrm{T}} \mathrm{S}_{\mathrm{i}}^{2} \mathrm{dt} \leq\right. \\
& \mathrm{S}^{\mathrm{T}}(0) \mathrm{G}_{0}^{-1}(\underline{\mathrm{x}}) \mathrm{S}(0)+\frac{1}{\eta_{\mathrm{w}}} \tilde{\mathrm{w}}^{\mathrm{T}}(0) \tilde{\mathrm{w}}(0) \\
& +\frac{1}{\eta_{\mathrm{m}}} \tilde{\mathrm{m}}^{\mathrm{T}}(0) \tilde{\mathrm{m}}(0)+\frac{1}{\eta_{\sigma}} \tilde{\sigma}^{\mathrm{T}}(0) \tilde{\sigma}(0) \\
& +\frac{1}{\eta_{\mathrm{w}_{\mathrm{r}}}} \tilde{\mathrm{w}}_{\mathrm{r}}^{\mathrm{T}}(0) \tilde{\mathrm{w}}_{\mathrm{r}}(0)+\sum_{\mathrm{i}=0}^{\mathrm{i}=\mathrm{n}} \frac{\beta_{\mathrm{i}}^{2}}{2} \int_{\mathrm{t}=0}^{\mathrm{T}} \mathrm{UD}_{\mathrm{i}}(\underline{\mathrm{x}})^{2} \mathrm{dt}
\end{aligned}
$$

If the system starts with initial conditions $\mathbf{S}=\mathbf{0}, \tilde{\mathbf{w}}=\mathbf{0}$, $\tilde{\mathbf{m}}(\mathbf{0})=\mathbf{0}, \tilde{\boldsymbol{\sigma}}(\mathbf{0})=\mathbf{0}, \tilde{\mathbf{w}}_{\mathrm{r}}(0)=0$ then the $\quad \mathrm{H}^{\infty}$ robust performance can be obtained as follows [20-21]:

$$
\sup _{\mathrm{UD}_{\mathrm{i}} \in \mathrm{L}_{2}[0, \mathrm{~T}]} \sum_{\mathrm{i}=1}^{\mathrm{i}=\mathrm{n}}\left(\frac{\left\|\mathrm{S}_{\mathrm{i}}\right\|}{\left\|\mathrm{UD}_{\mathrm{i}}\right\|} \leq \beta_{\mathrm{i}}\right)
$$

Where $\left\|S_{i}\right\|^{2}=\int_{t=0}^{T} S_{i}^{2} d t,\left\|U_{i}\right\|^{2}=\int_{t=0}^{T} U_{i}^{2} d t$ and $\beta_{i}$ is the prescribed attenuation level.

\section{Simulation AND RESUltS}

To verify the effectiveness of the proposed control system, a micro-motion stage powered by the linear piezoelectric motor (LPM) was used to investigate the stability and robustness of the system. The dynamic equation of LPM including hysteresis and stiffness behavior is described as follows $[10,18]$ :

$$
\ddot{\mathbf{x}}=-\frac{\mathbf{D}}{\mathbf{M}} \dot{\mathbf{x}}+\frac{\mathbf{K}}{\mathbf{M}} \mathbf{u}-\frac{\mathbf{F}_{\mathrm{H}}+\mathbf{F}_{\mathrm{L}}}{\mathbf{M}}
$$

where $\mathbf{x}$ stands for displacement of the $\mathrm{x}$-axis, $\mathrm{y}$-axis respectively; $\mathbf{M}$ is the effective mass of the moving stage; $\mathbf{D}$ is the friction coefficient; $\mathbf{F}_{\mathrm{L}}$ is external disturbance forces;
$\mathbf{K}$ is the voltage-to-force coefficient; $\mathbf{u}$ is the control volt of the LPMs; $\mathbf{F}_{\mathrm{H}}$ is the hysteresis frictional force given as below:

$$
\mathbf{F}_{\mathrm{H}}=\alpha \mathrm{b}+\delta|\dot{\mathbf{x}}|+\gamma \dot{\mathbf{x}}
$$

Where $\alpha, b, \delta, \gamma$ and $\mathrm{x}$ are parameters depending on the hysteresis loop and structure of the linear piezoelectric motor.

In general, the LPM is a complex and high non-linear system. According to the dynamic equation of the non-linear MIMO system (1), the dynamic equation of the LPM can be rewritten as follows:

$$
\ddot{\mathbf{x}}=-\frac{\mathbf{D}_{\mathrm{o}}}{\mathbf{M}_{\mathrm{o}}} \dot{\mathbf{x}}+\frac{\mathbf{K}_{\mathrm{o}}}{\mathbf{M}_{\mathrm{o}}} \mathbf{u}+\mathbf{U D}(\underline{\mathrm{x}})
$$

where $\mathbf{D}_{\mathrm{o}}, \mathbf{M}_{\mathrm{o}}$, and $\mathbf{K}_{\mathrm{o}}$ are nominal values of $\mathbf{D}, \mathbf{M}$ and $\mathbf{K}, \quad$ respectively. $\mathbf{U D}(\underline{\mathrm{x}})=-\frac{\mathbf{F}_{\mathrm{H} 0}+\mathbf{F}_{\mathrm{L} 0}}{\mathbf{M}_{0}}+\mathrm{f}\left(\mathbf{D}, \mathbf{F}_{\mathbf{H}}, \mathbf{F}_{\mathbf{L}}, \mathbf{K}, \mathrm{t}\right)$ denotes the uncertainties due to change in parameters, disturbances, and noises. This equation is used to build the simulation program for the proposed control system.

The initial parameters are used in simulation as follows:

$$
\begin{aligned}
& \mathrm{M}_{\mathrm{o}}=5 \mathrm{~kg} \mathrm{D}_{\mathrm{o}}=66 * 10^{-6}[\mathrm{~N} \cdot \mathrm{sec} / \mathrm{m}] \mathrm{K}_{\mathrm{Eo}}=3[\mathrm{~N} / \mathrm{Volt}], \\
& \eta_{\mathrm{w}}=\eta_{\mathrm{m}}=\eta_{\sigma}=\eta_{\mathrm{w}_{\mathrm{r}}}=0.01, \mathrm{n}=2, \mathrm{n}_{\mathrm{b}}=11, \mathrm{n}_{\mathrm{o}}=2, \mathrm{~K}=0.5, \\
& \beta=0.1 \text { and } 1 . \\
& \mathbf{w}=\left[\begin{array}{l}
0.1,0.1,0.1,0.1,0.1,0.1,0.1,0.1,0.1,0.1,0.1 \\
0.1,0.1,0.1,0.1,0.1,0.1,0.1,0.1,0.1,0.1,0.1
\end{array}\right], \\
& \mathbf{m}=\left[\begin{array}{l}
-1,-0.8,-0.6,-0.4,-0.2,0,0.2,0,4,0,6,0.8,1 \\
-1,-0.8,-0.6,-0.4,-0.2,0,0.2,0,4,0,6,0.8,1
\end{array}\right] \\
& \boldsymbol{\sigma}=\left[\begin{array}{l}
0.3,0.3,0,3,0.3,0.3,0,3,0.3,0.3,0,3,0.3,0.3 \\
0.3,0.3,0,3,0.3,0.3,0,3,0.3,0.3,0,3,0.3,0.3
\end{array}\right], \\
& \mathbf{w}_{\mathrm{r}}=\left[\begin{array}{l}
0.1,0.1,0.1,0.1,0.1,0.1,0.1,0.1,0.1,0.1,0.1 \\
0.1,0.1,0.1,0.1,0.1,0.1,0.1,0.1,0.1,0.1,0.1
\end{array}\right]
\end{aligned}
$$

The effects of uncertainties, external disturbances, and noise, $\mathbf{U D}(\underline{\mathbf{x}})$ was generated by a random signal with mean $=5$ and variance $=5$. Sample time $=0.01 \mathrm{~s}$.

The simulation results of the RRCMACS due to periodic step commands were represented in Fig. 3 for the $\mathrm{X}$-axis and the Fig. 4 for the Y-axis.

Fig. 5 and Fig. 6 represent the simulation results of the RARCMAC due to sinusoidal command in the $\mathrm{X}$-axis and the Y-axis, respectively. 

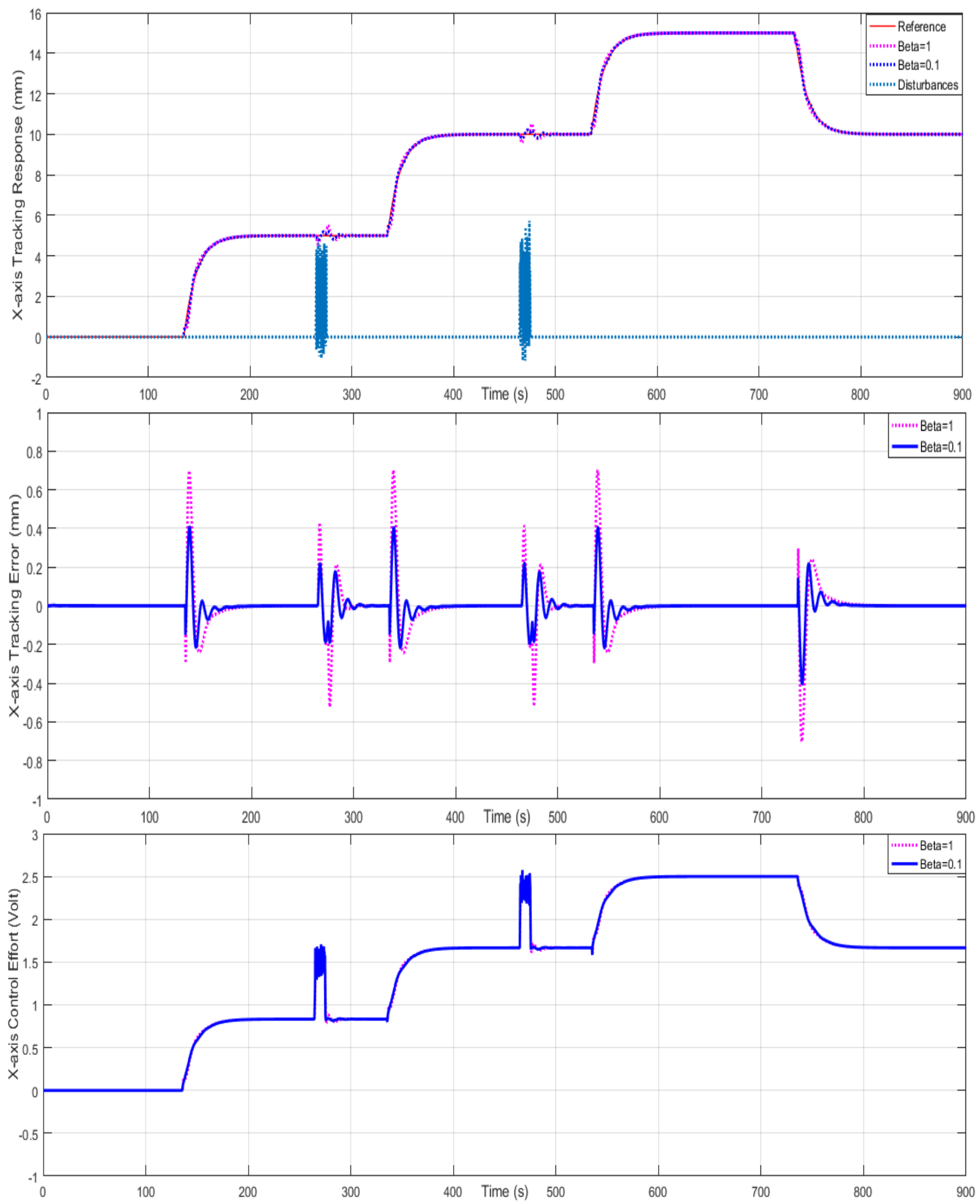

Fig. 3. Tracking Response, Tracking error and Control Effort of RRCMACS Due to Periodic Step Command in the X-Axis in Case of $\beta=0.1$ and $\beta=1$. 

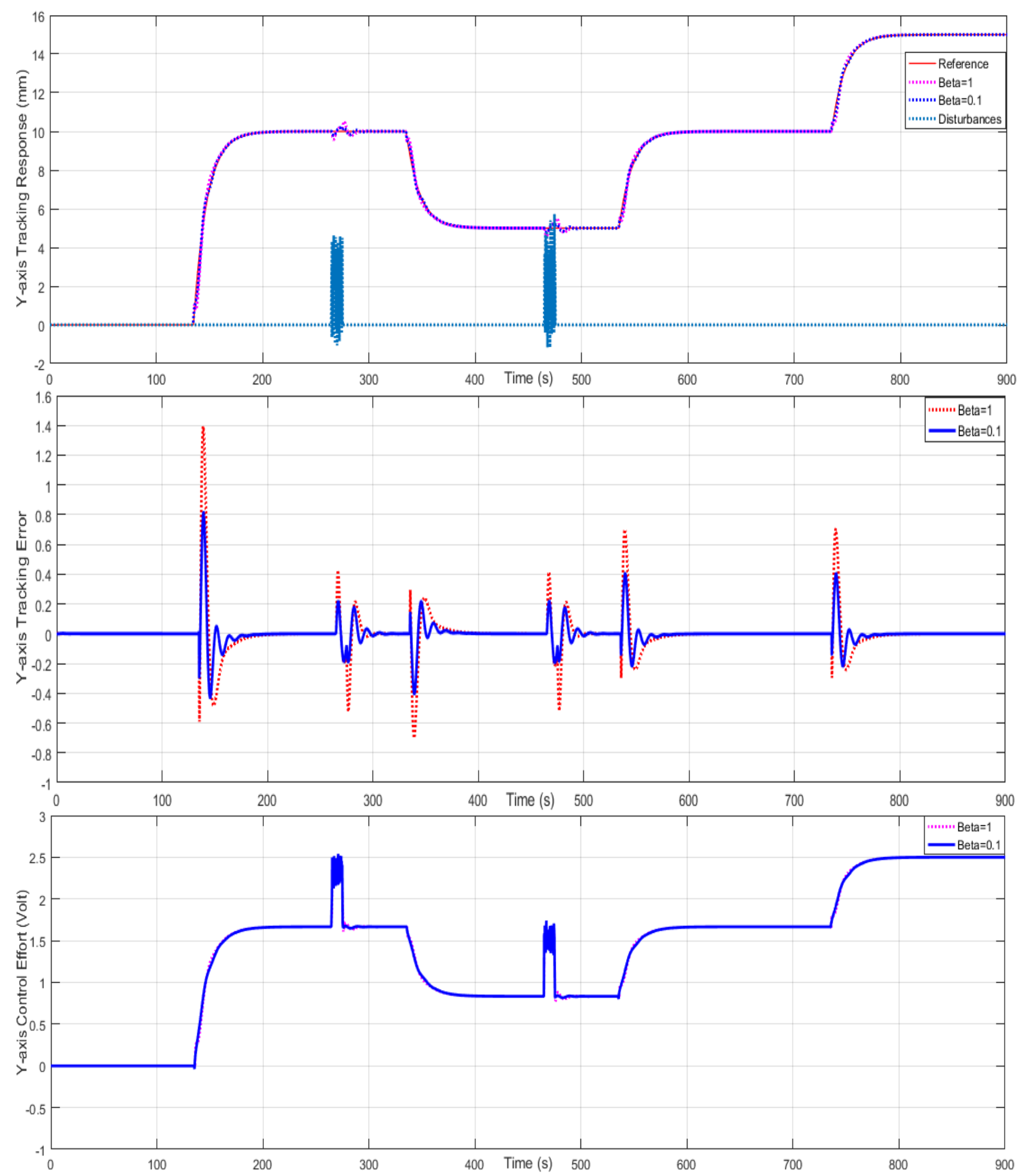

Fig. 4. Tracking Response, Tracking Error and Control Effort of RRCMACS Due to Periodic Step Command in the Y-Axis in Case of $\beta=0.1$ and $\beta=1$. 

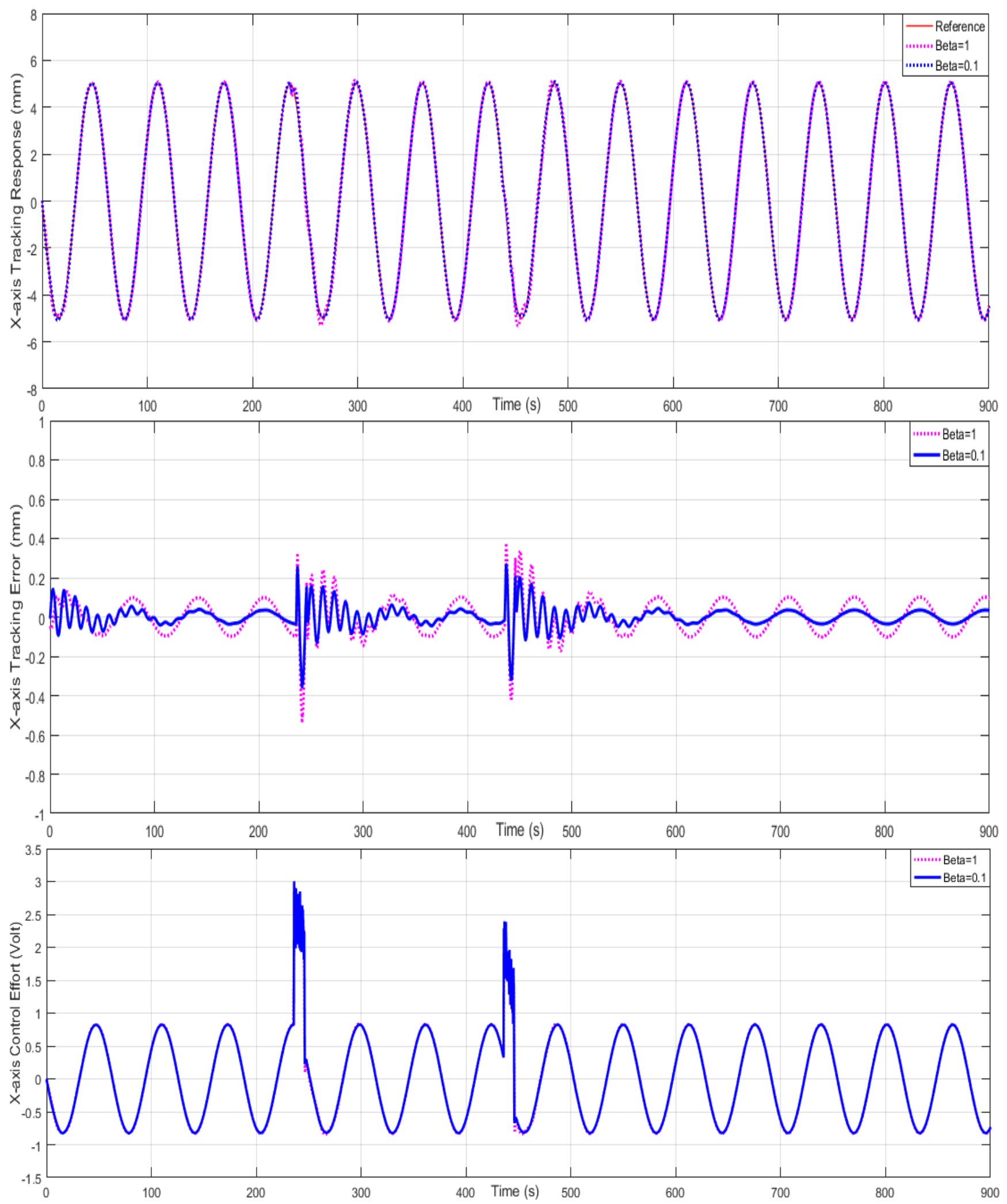

Fig. 5. Tracking Response, Tracking Error and Control Effort of RRCMACS Due to Sinusoidal Command in the X-Axis in Case of $\beta=0.1$ and $\beta=1$. 

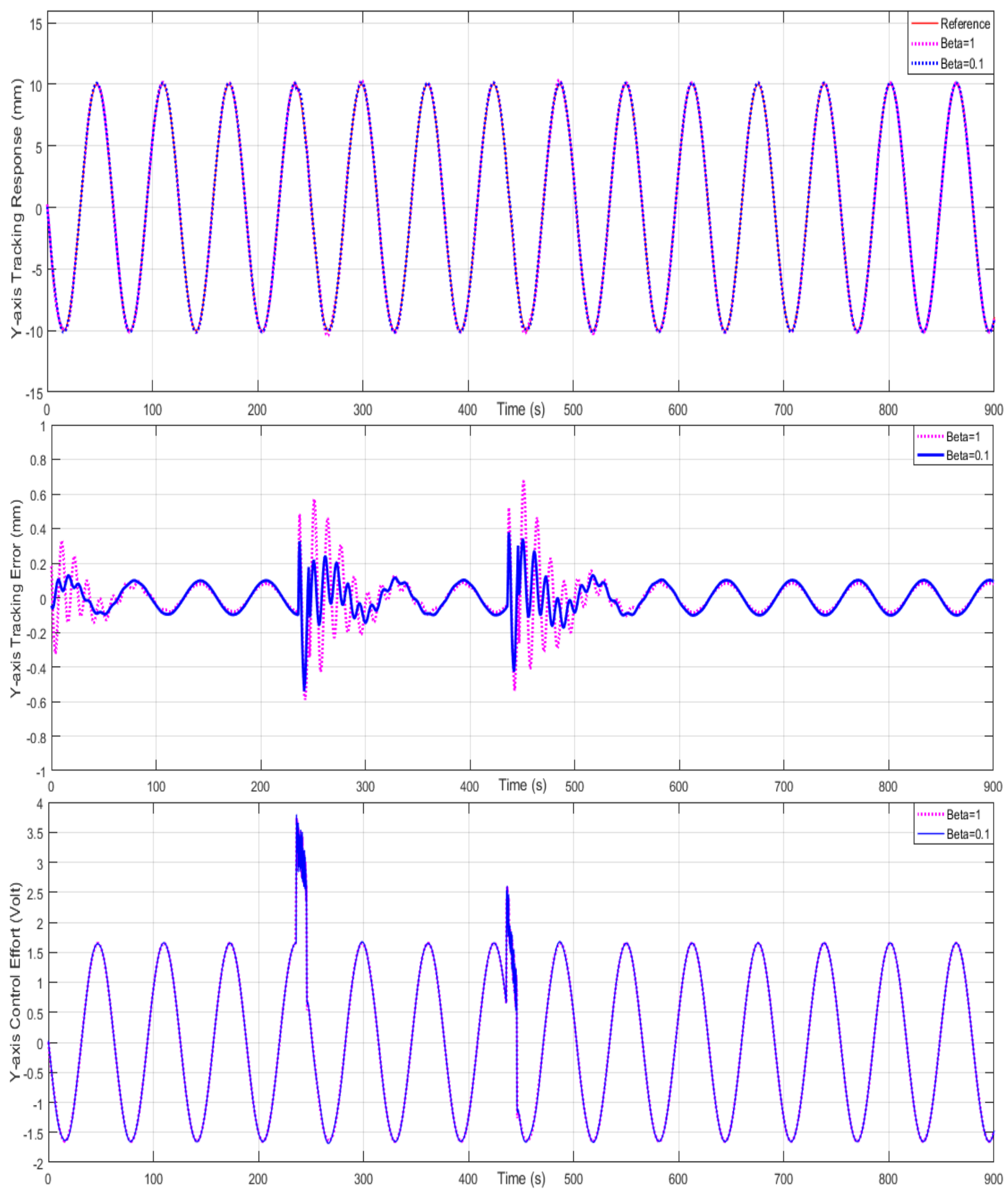

Fig. 6. Tracking Response, Tracking Error and Control Effort of RRCMACS Due to Sinusoidal Command in the Y-Axis in Case of $\beta=0.1$ and $\beta=1$. 
The simulation results showed that the RRCMACS achieved good tracking responses in the $\mathrm{X}$-axis and the $\mathrm{Y}$ axis. The control system obtained the stability and robustness in the presence of uncertainties, $\mathbf{U D}(\underline{\mathbf{x}})$ at $t_{1}=235 \mathrm{~s}$ to $\mathrm{t}_{2}=245 \mathrm{~s}$ and $\mathrm{t}_{3}=465 \mathrm{~s}$ to $\mathrm{t}_{4}=475 \mathrm{~s}$ for both periodic step command and sinusoidal command. The performance of the control system was better as the attenuation level of the $\mathbf{H}^{\infty}$ robust controller smaller.

\section{CONCLUSION AND FUTURE WORKS}

In this paper, the RRCMACS was proposed for the nonlinear MIMO system to achieve the stability and robustness in the presence of uncertainties, external disturbances, and noise, $\mathbf{U D}(\underline{\mathbf{x}})$. The proposed control system comprised the RCMAC and the $\mathrm{H}^{\infty}$ robust controller. Therein, the RCMAC was utilized to imitate the ideal sliding mode controller to minimize the error sliding manifold, and the $\mathrm{H}^{\infty}$ robust controller aims to attenuate the effects of uncertainties, external disturbance, and noise to the prescribed attenuation level. The simulation results of the LPM powered micromotion stage proved the effectiveness of the proposed control system. In addition, the $\mathbf{U D}(\underline{\mathbf{x}})$ stands for the inherent complex properties of the non-linear MIMO systems. Therefore, the proposed control system can handle other non-linear MIMO systems. However, this research needs to mention the responses of the hardware equipments to apply for the realtime control system.

\section{REFERENCES}

[1] Al-Ghanimi and J. Zheng, and Z. Man, A Fast Non-Singular Terminal Sliding Mode Control Based on Perturbation Estimation for Piezo Actuators Systems, International Journal of Control, 2017, vol.3. pp 122.

[2] J.S. Mo, Z.C. Qiu, J.Y. Wei, X.M. Zhang, Adaptive positioning control of an ultrasonic linear motor system, Robotics, and Computer-Integrated Manufacturing, 2017, vol.44, pp 156-173.

[3] Shuhui Bi, Lei Wang, Yongguo Zhao, and Mingcong Deng, Operatorbased Robust Control for Non-linear Uncertain Systems with Unknown Backlash-like Hysteresis, International Journal of Control, Automation and Systems, 2016, pp.469-477.

[4] Xinghua Zhang, Yantao Wang, and Xiaofei Fan, Stability Analysis of Linear Systems with An Interval Time-varying Delay - A Delay-rangepartition Approach, International Journal of Control, Automation and Systems, 2017, pp.518-526.

[5] V.Balaji, Dr. L.Rajaji, Shanthini K, Comparison Analysis of Model Predictive Controller with Classical PID Controller for $\mathrm{pH}$ Control Process Indonesian Journal of Electrical Engineering and Informatics (IJEEI), Vol. 4, No. 4, December 2016, pp. 250-255

[6] Tarun Varshney, Improved NN-PID control of MIMO systems with
PSO-based initialization of weights, Int. J. Automation and Control, Vol. 8, Nos. 2, 2014, pp. 158-172.

[7] Sundarapandian Vaidyanathan, Takagi-Sugeno fuzzy logic controller for Liu-Chen four-scroll chaotic system, Int. J. Intelligent Engineering Informatics, Vol. 4, No. 2, 2016, pp. 135-150.

[8] Garima Sinha, Pankaj Kumar Goswami, Sudhir Kumar Sharma, A Comparative Strategy Using PI \& Fuzzy Controller for Optimization of Power Quality Control, Indonesian Journal of Electrical Engineering and Informatics (IJEEI), Vol. 6, No. 1, 2018, pp. 118 124.

[9] G.Muni Reddy, T.Gowri Manohar, Fuzzy Logic Controller for Grid Connected Wind Energy Conversion System, Indonesian Journal of Electrical Engineering and Informatics (IJEEI), Vol. 6, No. 1,2018, pp. 37 44.

[10] A. Al-Ghanimi, J. Zheng, and Z. Man, A Fast Non-Singular Terminal Sliding Mode Control Based on Perturbation Estimation for Piezoelectric Actuators Systems. International Journal of Control, Vol 90, 2017, pp. 480-491.

[11] Abdelhak Msaddek, Abderraouf Gaaloul, and Faouzi M'sahli, Adaptive fuzzy supervision of the gain of the higher order sliding mode control, Int. J. Automation and Control, Vol. 9, Nos. 3, 2015, pp.228-246

[12] S. Jung, Stability Analysis of Reference Compensation Technique for Controlling Robot Manipulators by Neural Network, International Journal of Control, Automation and Systems, Vol. 15, no. 2, 2017, pp. 952-958.

[13] C. M. Lin and H. Y. Li, A novel adaptive wavelet fuzzy cerebellar model articulation control system design for voice coil motors', IEEE Trans. Ind.Electron, vol. 59, no. 4, 2012, pp. 2024-2033.

[14] C. M. Lin and H. Y. Li, TSK fuzzy CMAC-based robust adaptive backstepping control for uncertain non-linear systems, IEEE Trans. Fuzzy Syst., vol. 20, no. 6, 2012, pp. 1147-1154.

[15] Thanh Quyen Ngo, Ta Van Phuong, Robust Adaptive Self-Organizing Wavelet Fuzzy CMAC Tracking Control for Deicing Robot Manipulator, International Journal of Computers communication \& Control, Volt 10,2015, pp.567-578.

[16] S. Y. Wang, C. L. Tseng, and S. C. Chien, Adaptive fuzzy cerebellar model articulation control for switched reluctance motor drive, IET Elect.Power Appl., vol. 6, no. 3, 2012, pp. 190-202.

[17] V.P. Ta, X.K. Dang, and T.Q. Ngo, Adaptive Tracking Control Based On CMAC for Non-linear Systems, Proceedings of the International Conference on System Science and Engineering, 2017, pp 494-498.

[18] Van-Phuong Ta and Xuan-Kien Dang, An Innovative Recurrent Cerebellar Model Articulation Controller For Piezo-Driven Micromotion Stage, International Journal of Innovative Computing, Information and Control, Volume 14, Number 4, 2018, pp. 1527-1535.

[19] M. Bahita, Neural Stable Adaptive Control For a class of non-linear systems, Journal of Engineering Science and Technology, vol. 7, no. 1, 2012, pp. 97-18.

[20] P C Chen, C W Chen and W L Chiang, Linear Matrix Inequality Conditions of Nonlinear Systems by Genetic Algorithm-based $\mathrm{H}$ Adaptive Fuzzy Sliding Mode Controller, Journal of Vibration and Control, Jan 25, 2011.

[21] Alireza Alfi, Chaos suppression on a class of uncertain nonlinear chaotic systems using an optimal $\mathrm{H}$ infinity adaptive PID controller, Nonlinear Science, and Nonequilibrium and Complex Phenomena, Elsevier 2012. 